\author{
2022, Volume 9, ID 617 \\ DOI: $10.15342 / \mathrm{ijms} .2022 .617$
}

\title{
REVIEW
}

\section{Responses of Patients with T3-4N0-2M0 Rectal Cancer to Preoperative Chemoradiotherapy: Review of Current Literature}

\author{
Keita Mamady a,b,c (D), Bah Malick ${ }^{\text {b, c }}$, Kondano Saa Yawo ${ }^{\mathrm{c}}$, Camara Abou ${ }^{\mathrm{b}}$, Cisse Ibrahima Kalil ${ }^{\mathrm{b}}$, \\ Barry Maïmouna Sonna ${ }^{\mathrm{b}}$, Shen Wenbin ${ }^{\mathrm{a}}$ \\ 'Department of Radio-oncology, The Fourth Hospital, Hebei Medical University, Shijiazhuang 050011, China \\ "Surgical Oncology Unit of Donka University Hospital, Conakry 5575, Guinea \\ ${ }^{c}$ Faculty of Health Sciences and Techniques of Gamal Abdel Nasser University in Conakry, 10117 Guinea
}

\begin{abstract}
Objective: To investigate the efficacy and safety of chemoradiotherapy and radiotherapy followed by surgery in patients with locally advanced unresectable rectal cancer.

Material and method: We reviewed records for 65 patients with locally advanced unresectable rectal cancer treated by preoperative chemoradiotherapy or radiotherapy followed by surgery between 2013 and 2016. Of these, 23 patients were treated with preoperative chemoradiotherapy (40 - $45 \mathrm{~Gy}$ ) plus concomitant chemotherapy (5Fluorouracil + Calcium Folinate). For comparison, 42 similar patients treated by preoperative radiotherapy (45 - 50Gy) plus surgery served as control. The primary end-point of the study was overall survival and local control rate.

Results: No treatment plan was delayed because of toxicities in both groups. The radical resectability rate was $69.9 \%$ in the chemoradiotherapy group and $33.3 \%$ in the radiotherapy plus surgery group $(\mathrm{P}=0.024)$. The anal sphincter preservation rates were $26.6 \%$ and $3.7 \%$, respectively $(\mathrm{P}=0.028)$. The anal sphincter preservation rates of the lower rectal cancer were 27.3 $\%$ and $0.0 \%$, respectively $(\mathrm{P}=0.014)$. Response rates of chemoradiotherapy and radiotherapy plus surgery groups were 82.6 $\%$ and $61.9 \%(\mathrm{P}=0.053)$. The tumor downstage rates were $16(69.6 \%)$ and $24(57.1 \%)$ in these groups $(\mathrm{P}=0.206)$. The 3years overall survival rates were $66.7 \%$ and $55.6 \%(\mathrm{P}=0.485)$, and the tumor-free survival rates were $40.3 \%$ and $33.1 \%$ $(\mathrm{P}=0.663)$. The 3-years local recurrent rates were $26.9 \%$ and $48.1 \%(\mathrm{P}=0.174)$. No obvious late effects were found in either group.

Conclusion: The results of this study suggested at least that acute side effects of preoperative chemoradiotherapy can be tolerated, and a higher surgical resection rate can be achieved. However, the chemoradiotherapy did not improve the survival rate while it increased local recurrence due to the high rate of anal sphincter preservation. It is safe and effective to use 5Fluorouracil + Calcium Folinate and 5 - DFUR as a radiosensitizer during the whole course of radiotherapy.
\end{abstract}

KEYWORDS: Rectal cancer; Preoperative rectal cancer chemoradiotherapy; Chemoradiotherapy; Radiotherapy; Surgery.

Correspondence: Dr Mamady Keita, Address : Surgical Oncology Unit of Donka University Hospital, Conakry 5575, Guinea. Email: mamadykeita@ rocketmail.com

Copyright (C) 2022 Mamady $\mathbf{K}$ et al. This is an open access article distributed under the Creative Commons Attribution 4.0 International, which permits unrestricted use, distribution, and reproduction in any medium, provided the original work is properly cited.

\section{INTRODUCTION}

The curative effect of adjuvant radiotherapy (RT) for rectal cancer is a concern. Before 2000, RT for rectal cancer in China is mostly limited to postoperative treatment, and there are few reports on preoperative RT. Nowadays, there is no large sample study on its influence on the local recurrence rate, and preoperative RT in rectal cancer is not widely accepted in the entire surgical field because of the lack of support from the results of large clinical randomized controlled studies. At the same time, for patients with T3-4N0-2 M0 rectal cancer, preoperative RT or concomitant chemoradiotherapy (CRT) may be used to achieve the possible purpose of total mesorectal resection. Most clinical studies of preoperative CRT are based on platinum regimens to obtain the radiosensitizing effect. [15] This article retrospectively analyzes the efficacy and toxicity of preoperative CRT in patients with locally advanced unresectable rectal cancer. 


\section{MATERIALS AND METHODS}

Patient selection criteria

Patients who met the following criteria were included in this study: (1) Histologically confirmed rectal carcinoma; (2) No history of cancer treatment; (3) No previous malignancies; (4) Complete clinical data; (5) Tumors were found to be located in middle or lower rectal by endoscopy. Patients who had been previously treated with surgery were excluded. A total of 150 consecutive patients with rectal cancer were treated with pre-operative CRT or RT followed by surgery at a single institution (The Fourth Affiliated Hospital of Hebei Medical University, Shijiazhuang, China) between January 1, 2013, and December 31, 2016. Among them, 85 patients did not meet our inclusion criteria.

Patients clinical characteristics

A total of 23 patients with pathologically confirmed rectal cancer were set as the preoperative CRT group, including 17 males and 6 females, aged $36-78$ years, with a median age of 58 years. There were 7 cases of moderately differentiated tubular adenocarcinoma, 4 cases of moderately differentiated adenocarcinoma, 4 cases of poorly differentiated adenocarcinoma, 6 cases of mixed adenocarcinoma, and 2 cases of papillary adenocarcinoma. There were 42 cases in the control group, 33 males and 9 females, aged $22-81$ years old, with a median age of 61 years. The pathological types included 14 cases of moderately differentiated tubular adenocarcinoma, 9 cases of moderately differentiated adenocarcinoma, 5 cases of poorly differentiated adenocarcinoma, 11 cases of mixed adenocarcinoma, and 3 cases of highly differentiated adenocarcinoma (Table 1). The Karnofsky performance status in each group was $\geq 70 \%$. The digital rectal examination and imaging (abdominal and pelvic ultrasound, CT or MRI) allow preoperative staging and confirmation of unresectable rectal tumors. All patients were staged according to the American Joint Commission (AJCC) 7th edition criteria [6]. The initial staging consisted of a history and medical examination, routine blood tests, endoscopy, and a chest computed tomography (CT). Thirty- two patients had PET-CT before treatments. The TNM staging of colorectal cancer results are as follows: preoperative CRT group included 7 cases in T3 NOM0 stage, 11 cases in T4 NOM0 stage, and 5 cases in the T4N1M0 stage; in the control group, 21 cases in the T3N0M0 stage, 6 cases in stage T3N1M0, 4 cases in stage T4N0M0, 7 cases in stage T4N1M0, and 4 cases in stage T4N2M0. For all patients, the tumors were all located in the middle, and lower rectum $(6-12 \mathrm{~cm})$, and the distance between the lower edge of the tumor and the anus was ( 7 . $34 \pm 4.15) \mathrm{cm}$ and $(7.19 \pm 3.21) \mathrm{cm}$, respectively, the two groups are similar. There were 11 cases and 20 cases of low rectal cancer $(8 \mathrm{~cm}$ from the anal margin). At diagnosis, a minimum of 3 or more clinical symptoms was present such as anal pain, the blood in the stool, abdominal distension, increased stool frequency, soreness in the lumbosacral area, and anal mass.

Table 1 Characteristics of 65 locally advanced rectal cancer patients [ cases (\%)]

\begin{tabular}{|c|c|c|c|}
\hline \multirow{3}{*}{ Patients characteristics } & $\begin{array}{l}\text { Whole } \\
\text { group }\end{array}$ & $\begin{array}{c}\text { CRT+ Surgery } \\
\text { group }\end{array}$ & RT + Surgery group \\
\hline & $n=65$ & $n=23$ & $n=42$ \\
\hline & No. $(\%)$ & No. $(\%)$ & No. $(\%)$ \\
\hline Age, median (range, years) & $58(22-81)$ & $56(36-78)$ & $61(22-81)$ \\
\hline \multicolumn{4}{|l|}{ Sexe } \\
\hline - $\quad$ Male & $50(76.9)$ & $17(73.9)$ & $33(78.6)$ \\
\hline - Female & $15(23.1)$ & $6(26.1)$ & $9(21.4)$ \\
\hline \multicolumn{4}{|l|}{ Primary site } \\
\hline - Low rectum & $31(47.7)$ & $11(47.8)$ & $20(47.6)$ \\
\hline - $\quad$ Middle rectum & $34(52.3)$ & $12(52.2)$ & $22(52.4)$ \\
\hline \multicolumn{4}{|l|}{ TNM - stage } \\
\hline - T3N0M0 & $28(43.1)$ & $7(30.4)$ & $21(50.0)$ \\
\hline $\begin{array}{ll}- & \text { T3N1M0 } \\
\end{array}$ & $6(9.2)$ & $0(0.00)$ & $6(14.3)$ \\
\hline - T4N0M0 & $15(23.1)$ & $11(47.8)$ & $4(9.5)$ \\
\hline - $\quad$ T4N1M0 & $12(18.5)$ & $5(21.7)$ & 7 (16.7) \\
\hline - $\quad$ T4N2M0 & $4(6.1)$ & $0(0.00)$ & $4(9.5)$ \\
\hline \multicolumn{4}{|l|}{ Histology } \\
\hline - Adenocarcinoma & $25(38.5)$ & $8(34.8)$ & $17(40.5)$ \\
\hline - $\quad$ Tublar adenocarcinoma & $21(32.3)$ & $7(30.4)$ & $14(3.33)$ \\
\hline - $\quad$ Mixed adenocarcinoma & $17(26.2)$ & $6(26.1)$ & $11(26.2)$ \\
\hline - $\quad$ Papillary adenocarcinoma & $2(3.1)$ & $2(8.7)$ & $0(0.00)$ \\
\hline \multicolumn{4}{|l|}{ Grades } \\
\hline - Hihgly differentiated & $3(4.6)$ & $0(0.00$ & $3(7.1)$ \\
\hline - $\quad$ Moderately differentiated & $34(52.3)$ & $11(47.8)$ & $23(54.8)$ \\
\hline - $\quad$ Poorly differentiated & $9(13.8)$ & $4(17.4)$ & $5(11.9)$ \\
\hline$-\quad \mathbf{N S}$ & $19(29.2)$ & $8(34.8)$ & $11(26.2)$ \\
\hline \multicolumn{4}{|l|}{ Tumor response (RECIST) } \\
\hline - $\quad$ Complete response & $4(6.2)$ & $4(17.3)$ & $0(0.00)$ \\
\hline - Partial response & $41(63.1)$ & $15(65.2)$ & $26(61.9)$ \\
\hline - $\quad$ Stable disease & $20(30.8)$ & $4(17.3)$ & $16(38.1)$ \\
\hline - $\quad$ Progressive disease & $0(0.00)$ & $0(0.00)$ & $0(0.00)$ \\
\hline
\end{tabular}


Radiotherapy target area definition

The RT field was similar in both groups. All use 10MV Xray, radiation fields included anteroposterior pelvic fields plus two horizontal lateral fields. In some cases, increased radiation boost dose after $40 \mathrm{~Gy}$ to the local tumor. The external beam radiotherapy (EBRT) dose in the preoperative CRT group was DT 40 - $46 \mathrm{~Gy}$, with an average of $41.5 \mathrm{~Gy}, 20$ to 23 fractions, 4 to 5 weeks. The dose in the control group was DT40-50 Gy, with an average of $42.6 \mathrm{~Gy}, 20-25$ fractions, completed in 4-5 weeks.

Chemotherapy

The chemotherapy regimen in the preoperative CRT group was based on Calcium Folinate (CF, $300 \mathrm{mg}$ ), 5Fluorouracil (5- FU, $500 \mathrm{mg} / \mathrm{m} 2$ ), on the $\mathrm{d} 1$, d2, and $\mathrm{d} 3$ of the first week and the $\mathrm{d} 1-\mathrm{d} 2$, and $\mathrm{d} 3$ of the 4 th week. For the remainder of the RT period, they received $200 \mathrm{mg}$ deoxyfluridine (5 - DFUR) orally, three times/day. After completing CRT or RT, preoperative imaging study and surgery were performed 4 to 5 weeks later, and conventional postoperative chemotherapy was based on 5 - FU up to 4 to 6 cycles.

Adverse reaction evaluation criteria

According to the requirements of the World Health Organization (WHO), acute toxicity was evaluated in the two groups.

Follow- up and efficacy evaluation

Patients were followed up via physical examination, chest and abdominal CT, and coloscopy six months later. The date of the last follow-up was December 31, 2017. The preoperative curative effect is divided into complete remission (CR), partial remission (PR), stable (SD), and disease progression (PD) according to the standards of the RECIST criteria for solid tumors [7]. Summarize the effect of surgical treatment (radical resection rate, anal sphincter preservation rate, and surgical complications); Compare the pre-treatment clinical staging and the pathological staging of the excised specimens, and comprehensively evaluate the short-term curative effect and down-stage effect. All cases were followed up for 3-years, and the 3year survival rate and local recurrence rate of the two groups were compared.

Statistical analysis

An analysis of the baseline characteristics was carried out in both groups (Table 1). A descriptive analysis was performed using mean (with range) and proportions with the data. The overall survival was measured from the date of rectal cancer diagnosis to death from any cause or the last known follow-up date. Patients were considered to be experiencing local failure only if histologic evidence was observed in the primary tumor. Lymph nodes metastases were diagnosed based on the appearance of new nodes in regions where no enlarged nodes had been identified before irradiation. Suspected regional lymph node recurrences were confirmed by CT- scan or MRI/ ultrasound of abdomen and pelvis. The survival rate and local control rate were calculated using the Kaplan Meier method, and the Log-rank test was used to evaluate the difference between different the two groups. All statistical computations were done using SPSS 21.0 (SPSS Inc, Chicago, IL). P-value $<0.05$ was considered statistically significant.

\section{RESULTS}

In the preoperative CRT group, 7 - 15 days (median 8 days) after the start of treatment, and 9-18 days (median 10 days) in the RT group, the clinical symptoms were improved to varying degrees, such as reduced mucus or blood and mucus in the stool, reduced abdominal distension or local pain; the tumor palpable by digital rectal examination began to shrink, and the blood staining of the finger cuff was significantly reduced. In the preoperative CRT group, there were 16 cases of radical resection, 5 cases of palliative operation, and 2 cases of exploratory operation; in the control group, 14 cases of radical operation, 24 cases of palliative operation, and 4 cases of exploratory operation. The rates of radical resection were $69.6 \%$ and $33.3 \%$, respectively $(\chi 2=5.08$, $\mathrm{P}=0.024)$. In the two groups, $26.6 \%$ and $3.7 \%$ of the cases retained anal sphincter function $(\chi 2=4.85, \mathrm{P}=0.028)$. Among them, the anal sphincter preservation rates of low rectal cancer were $27.3 \%$ and $0.0 \%(\chi 2=6.04, \mathrm{P}=0.014)$. Patients in the two groups died without surgical complications. Pathological changes of surgery specimens: preoperative CRT group had 3 cases of thirddegree reaction, 15 cases of second-degree reaction, 5 cases of first-degree reaction; the control group had 27 cases of second-degree reaction and 15 cases of firstdegree reaction. Combining clinical and imaging examination and surgical pathology results to evaluate the efficacy: the preoperative chemoradiotherapy group had CR 4 cases, PR 15 cases, and SD 4 cases; the control group had PR 26 cases and SD 16 cases. The total remission rate was $82.6 \%$ and $61.9 \%$, respectively $(\chi 2=3.94, \mathrm{P}=$ $0.043)$. In the whole group, there was no change in $\mathrm{N}$ staging. In contrast, the TNM staging after treatment in the two groups was: 4 cases in the TONOM0 stage, 11 cases in the T3N0M0 stage, 3 cases in the T3N1M0 stage, 2 cases in the T4N0M0 stage, and 3 cases in the T4N1M0 stage in the preoperative chemoradiotherapy group. The control group: 4 cases in the T2NOM0 stage, 6 cases in the T2N1M0 stage, 16 cases in the T3N0M0 stage, 3 cases in the T3N1M0 stage, 9 cases in the T4N1M0 stage, 4 cases in the T4N2M0 stage. In the preoperative CRT group, 4 cases of $\mathrm{T} 3$ were reduced to $\mathrm{T} 0$, and 12 cases of $\mathrm{T} 4$ were reduced to $\mathrm{T} 3$; in the control group, 17 cases of $\mathrm{T} 3$ were reduced to $\mathrm{T} 2$, and 7 cases of $\mathrm{T} 4$ were reduced to $\mathrm{T} 3$. The rate of decline in $\mathrm{T}$ stage was $69.6 \%$ and $57.1 \%$, respectively $(\chi 2=1.60, \mathrm{P}=0.206)$.

The 3-year survival rates of the preoperative CRT group and the control group were $66.7 \%$ and $55.6 \%\left(\chi^{2}=0.49\right.$, $\mathrm{P}=0.485$ ), and the 3 -years tumor-free survival rates were 40.3 and $33.1 \%$, respectively. $33.2 \%(\chi 2=0.87, \mathrm{P}=0$. $663)$, the local recurrence rates were $26.9 \%$ and $48.1 \%\left(\chi^{2}\right.$ $=1.85, \mathrm{P}=0.174)$. In the preoperative CRT group, 1 of the 4 anus-preserving patients survived with metastatic tumors in both lungs, and the remaining 3 patients did not find local recurrence or distant metastasis. The defecation function of the 4 anus-preserving patients was normal. The acute toxicity of the preoperative CRT group was mainly anal skin reaction. Nine patients developed third-degree anal skin reactions and were given local symptomatic treatment; three patients developed third-degree diarrhea and improved after 3 days of oral administration of Loperamide 2mg; Two patients had a third-degree 
leukopenia and were given a subcutaneous injection of Filgrastim $75 \mu \mathrm{g} /$ days, after 3 days, the leukocytes reached to $5.5 \times 109 / \mathrm{L}$. The rest of the patients had no serious reactions and were not given special treatment. In the control group, there were 10 cases of third-degree anal skin reaction, 2 cases of third-degree diarrhea, and 2 cases of third-degree leukopenia. The acute toxicity of preoperative CRT group and control group was $60.9 \%$ and $33.3 \%(\chi 2=$ $3.25, \mathrm{P}=0.071)$. None of the patients in the two groups interrupted or discontinued treatment due to acute side effects, and no serious late-stage reactions that could affect the quality of life were found.

\section{DISCUSSION}

A study from the MD Anderson Cancer Center in the United States shows that preoperative simultaneous chemotherapy plus RT for locally advanced rectal cancer can better control pelvic lesions and improve survival than preoperative RT. In contrast, the acute toxicity of preoperative CRT is relatively large, but it can be tolerated, and the late side effects are no different from RT alone $[1,2,3]$. In this study, the incidence of grade III and IV acute toxicity in the preoperative CRT group was higher than in the control group. Still, the difference was not significant, and after symptomatic and supportive treatment, it did not affect treatment progress. No serious late-stage side effects that affect the quality of life were found in the two groups. The complications of preoperative RT for locally advanced rectal cancer plus simultaneous low-dose 5 - FU continuous perfusion are acceptable. The purpose is to achieve a certain clinical and pathological downstage effect of the tumor and increase the radical resection rate. Increasing the resection rate will play a certain role in improving the local control rate and survival rate $[4,5]$.

In most clinical studies, 5 - FU was given by continuous intravenous infusion during the whole course of RT or intravenous infusion in the first or last week of RT [1- 3], or with 5- FU or calcium folinate is taken orally throughout the entire course as a radiosensitizer [ 7 - 11].

To improve the effectiveness of local RT to relieve the clinical symptoms of patients with locally advanced rectal cancer as soon as possible and improve the effect of systemic treatment, patients in the CRT group received 3 days of intravenous chemotherapy in the first week and last week of radiotherapy. At the same time, 5 - DFUR was added orally as a radiosensitizer during all RT courses. Tomoda et al. [12] studied 53 cases of advanced colorectal carcinoma. They found that a single oral administration of 5 - DFUR significantly increased the concentration of 5 FU in tumor tissues and lymph nodes, and the effect of pyrimidine nucleoside phosphorylase was observed. Activation, the tumor tissue at $24 \mathrm{~h}$, and the lymph node at $8 \mathrm{~h}$ can still maintain an effective 5- FU concentration. The activity of pyrimidine nucleoside phosphorylase in tumor tissues is significantly higher than that in the normal intestinal mucosa $(\mathrm{P}<0.05)$, and 5 - DFUR kills tumor cells in the primary tumor and lymph node through the transformation of pyrimidine nucleoside phosphorylase.
Therefore, it should be safe and effective to use the appropriate dose of 5 - DFUR as a radiosensitizer during the whole course of RT, and it should be safe and effective to choose oral drugs as a radiosensitizer instead of continuous intravenous infusion of $5-\mathrm{FU}$ during RT course, which can significantly reduce the occurrence of phlebitis. Moreover, it's convenient and easy to perform, easy for patients to accept, and can be performed in outpatient clinics. Of course, the optimal dose of oral 5 DFUR during concurrent CRT needs to be further explored.

In this study, the preoperative CRT group had a higher anal sphincter preservation rate than the control group, and it was significant. Therefore, although the local tumor control rate and the survival rate of the preoperative CRT group are higher than that of the control group, there was no significant significance, but it can at least explain that the CRT group did not increase the local recurrence and reduced the survival rate due to the reduction of the scope of surgery by the anal sphincter preservation. This is of great significance to meet the patient's requirements for anal preservation and improve the patient's quality of life. Getting the highest possible surgical resection rate through preoperative chemoradiotherapy is the key to a cure $[7,11$ - 14]. In this study, the surgical resection rate of the preoperative concurrent CRT group was significantly higher than that of the control group, and it was significant, while the results of higher survival rate and local control rate were not significant. This may be related to the small number of cases in our study, and further investigation with more cases is expected in clinical practice. As far as RT technology is concerned, it should be emphasized to formulate precise RT plans to minimize the toxic and side effects of normal tissues. Because the results of the biological model analysis show that the three-field and four-field techniques have fewer intestinal complications than the two-field technique $[15,16]$, this issue should be paid more attention to when we perform concurrent CRT.

\section{ACKNOWLEDGMENTS}

None.

\section{AUTHORS' CONTRIBUTIONS}

The participation of each author corresponds to the criteria of authorship and contributorship emphasized in the Recommendations for the Conduct, Reporting, Editing, and Publication of Scholarly work in Medical Journals of the International Committee of Medical Journal Editors. Indeed, all the authors have actively participated in the redaction, the revision of the manuscript, and provided approval for this final revised version.

\section{COMPETING INTERESTS}

The author declare no competing interests with this case.

\section{FUNDING SOURCES}

None. 


\section{REFERENCES}

[1] Bitterman DS, Resende-Salgado L, Moore HG, Sanfilippo NJ, Gu P, Hatzaras I, et al. Predictors of Complete Response and Recurrence Following Neoadjuvant Chemoradiation Therapy in Rectal Cancer. Radiation Oncol Biol Physic. 2015; 93 (3): E145-E146. DOI: 10.1016/j.ijrobp.2015.07.919

[2] Callegaro D, Miceli R, Brunelli C, Colombo C, Sanfilippo R, Radaelli $\mathrm{S}$, et al. Long-term morbidity after multivisceral resection for retroperitoneal sarcoma, Br J Surg. 2015 Aug; 102(9): 1079-1087. DOI: $10.1002 /$ bjs. 9829

[3] De Caluwe L, Van Nieuwenhove Y, Ceelen WP. Preoperative chemoradiation versus radiation alone for stage II and III resectable rectal cancer. Cochrane Database Syst Rev. $2013 \quad$ Feb $28 \quad ;(2): \quad$ CD006041. DOI: $10.1002 / 14651858 . c d 006041 . p u b 3$

[4] Jin F, Luo H, Zhou J, Wu Y, Sun H, Liu H, et al. Dose-time fractionation schedules of preoperative radiotherapy and timing to surgery for rectal cancer. Ther Adv Med Oncol. 2020 Feb 29; 12:1758835920907537. DOI: $10.1177 / 1758835920907537$

[5] Valentini V, Coco C, Cellini N, Picciocchi A, Fares MC, Rosetto ME, et al. Ten years of preoperative chemoradiation for extraperitoneal T3 rectal cancer: acute toxicity, tumor response, and sphincter preservation in three consecutive studies. Int J Radiat Oncol Biol Phys. 2001 Oct 1; 51(2):37183. DOI: $10.1016 / \mathrm{s} 0360-3016(01) 01618-2$

[6] Edge SB, Compton CC. The American Joint Committee on Cancer: the 7th edition of the AJCC cancer staging manual and the future of TNM. Ann Surg Oncol. 2010 Jun; 17(6):1471-4. DOI: $10.1245 / \mathrm{s} 10434-010-0985-4$

[7] Eisenhauer EA, Therasse P, Bogaerts J, Schwartz LH, Sargent D, Ford R, et al. New response evaluation criteria in solid tumors: revised RECIST guideline (version 1.1). Eur J Cancer. 2009 Jan; 45(2):228-47. DOI: $10.1016 /$ j.ejca.2008.10.026

[8] Gérard JP, Chamorey E, Gourgou-Bourgade S, Benezery K, de Laroche G, Mahé MA, et al. Clinical complete response (cCR) after neoadjuvant chemoradiotherapy and conservative treatment in rectal cancer. Findings from the ACCORD 12/PRODIGE 2 randomized trial. Radiother Oncol. 2015 May; 115(2):246-52. DOI: 10.1016/j.radonc.2015.04.003

[9] Fucini C, Pucciani F, Elbetti C, Gattai R, Russo A. Preoperative radiochemotherapy in $\mathrm{t} 3$ operable low rectal cancers: a gold standard? World J Surg. 2010 Jul;34(7):160914. DOI: $10.1007 / \mathrm{s} 00268-010-0513-5$

[10] Cellier P, Leduc B, Martin L, Vié B, Chevelle C, Vendrely $\mathrm{V}$, et al. Phase II study of preoperative radiation plus concurrent daily tegafur-uracil (UFT) with leucovorin for locally advanced rectal cancer. BMC Cancer. 2011 Mar 16; 11:98. DOI: $10.1186 / 1471-2407-11-98$

[11] Nakagawa R, Inoue Y, Ohki T, Kaneko Y, Maeda F, Yamamoto M. Efficacy and short-term outcomes of preoperative chemoradiotherapy with intermittent oral tegafur-uracil plus leucovorin in Japanese rectal cancer patients: a single center experience retrospective analysis. World J Surg Oncol. 2017 May 31; 15(1):112. DOI: $10.1186 / \mathrm{s} 12957-017-1177-5$

[12] Tomoda H, Morita M, Matsuoka H, Baba H, Taketomi A, Kohnoe S, et al. [Pyrimidine nucleoside phosphorylase activity, 5-fluorouracil concentration and thymidylate synthase inhibition rate in colorectal cancer after oral administration of 5'-doxifluridine]. Gan To Kagaku Ryoho. 1997 Jun;24(8):971-4

[13]Zheng JF, Wang HD. 5-Fluorouracil concentration in blood, liver and tumor tissues and apoptosis of tumor cells after preoperative oral 5'-deoxy-5-fluorouridine in patients with hepatocellular carcinoma. World J Gastroenterol. $2005 \mathrm{Jul} 7$; 11(25):3944-3947. DOI: 10.3748/wjg.v11.i25.3944

[14] Pucciarelli S, Toppan P, Friso ML, Russo V, Pasetto L, Urso $\mathrm{E}$, et al. Complete pathologic response following preoperative chemoradiation therapy for middle to lower rectal cancer is not a prognostic factor for a better outcome. Dis Colon Rectum. 2004 Nov; 47(11):1798-807. DOI: $10.1007 / \mathrm{s} 10350-004-0681-1$

[15] Wiegering A, Isbert C, Dietz UA, Kunzmann V, Ackermann $\mathrm{S}$, Kerscher A, et al. Multimodal therapy in treatment of rectal cancer is associated with improved survival and reduced local recurrence - a retrospective analysis over two decades. BMC Cancer. 2014 Nov 6; 14:816. DOI: $10.1186 / 1471-2407-14-816$

[16] Birgisson H, Påhlman L, Gunnarsson U, Glimelius B; Swedish Rectal Cancer Trial Group. Adverse Effects of Preoperative Radiation Therapy for Rectal Cancer: LongTerm Follow-Up of the Swedish Rectal Cancer Trial. J Clin Oncol. 2005 Dec 1; 23(34):8697-705. DOI: $10.1200 /$ jco.2005.02.9017 